\title{
UV effects on the primary productivity of picophytoplankton: biological weighting functions and exposure response curves of Synechococcus
}

\author{
P. J. Neale, A. L. Pritchard, and R. Ihnacik \\ Smithsonian Environmental Research Center, Edgewater, MD, USA \\ Correspondence to: P. J. Neale (nealep@ si.edu) \\ Received: 9 October 2013 - Published in Biogeosciences Discuss.: 11 December 2013 \\ Revised: 25 March 2014 - Accepted: 2 April 2014 - Published: 28 May 2014
}

\begin{abstract}
A model that predicts UV effects on marine primary productivity using a biological weighting function (BWF) coupled to the photosynthesis-irradiance response (BWF/P-E model) has been implemented for two strains of the picoplanktonic cyanobacteria Synechococcus, WH7803 and WH8102, which were grown at two irradiances (77 and $174 \mu \mathrm{mol} \mathrm{m}^{-2} \mathrm{~s}^{-1}$ photosynthetically available radiation (PAR)) and two temperatures $\left(20\right.$ and $\left.26^{\circ} \mathrm{C}\right)$. The model was fit using photosynthesis measured in a polychromatic incubator with 12 long-pass filter configurations with $50 \%$ wavelength cutoffs ranging from 291 to $408 \mathrm{~nm}$, giving an effective wavelength range of $280-400 \mathrm{~nm}$. Examination of photosynthetic response vs. weighted exposure revealed that repair rate progressively increases at low exposure but reaches a maximum rate above a threshold exposure (" $E_{\max }$ "). Adding $E_{\max }$ as a parameter to the BWF/P-E model provided a significantly better fit to Synechococcus data than the existing " $E$ " or " $T$ " models. Sensitivity to UV inhibition varied with growth conditions for both strains, but this was mediated mainly by variations in $E_{\max }$ for WH8102 while both the $\mathrm{BWF}$ and $E_{\max }$ changed for WH7803. Higher growth temperature was associated with a considerable reduction in sensitivity, consistent with an important role of repair in regulating sensitivity to UV. Based on nominal water column conditions (noon, solstice, $23^{\circ}$ latitude, "blue" water), the $\mathrm{BWF}_{E_{\max }} / \mathrm{P}-\mathrm{E}$ model estimates that UV + PAR exposure inhibits Synechococcus photosynthesis from 78 to $91 \%$ at $1 \mathrm{~m}$, and integrated productivity to $150 \mathrm{~m} 17-29 \%$ relative to predicted rates in the absence of inhibition.
\end{abstract}

\section{Introduction}

Inhibition of phytoplankton photosynthesis by solar ultraviolet (UV) and photosynthetically available radiation (PAR) occurs at least episodically in almost all near-surface waters of global marine and freshwater environments (Villafañe et al., 2003; Harrison and Smith, 2009). However, most detailed studies of the spectral dependence of inhibition in marine phytoplankton have focused on species or assemblages characteristic of high-latitude polar environments where the UVB $(280-320 \mathrm{~nm})$ spectrum has been (and continues to be) affected by seasonally severe depletion of stratospheric ozone (Weatherhead and Andersen, 2006). Hence, little is known about the spectral dependence of photosynthetic response for phytoplankton prevalent in the central mid- and low-latitude ocean. Important components of the assemblages in these regions are strains of picoplanktonic cyanobacteria under the broad taxonomic classification Synechococcus sp. - major contributors to global primary production. Synechococcus sp. inhabit a wide range of temperate and subtropical environments having moderate to high UV transparency (Fichot and Miller, 2010; Garczarek et al., 2008). Although there is ample evidence from laboratory studies that the photosynthetic apparatus of Synechococcus is impaired by UV radiation (e.g., Garczarek et al., 2008; Mella-Flores et al., 2012; Six et al., 2007b), it is not clear how much these effects result in decreased photosynthetic performance under natural conditions. 
Definition of the spectral dependence of inhibition in Synechococcus sp. is motivated by several reasons. First, efficient numerical approaches have been developed enabling the inclusion of spectrally dependent inhibition in the prediction of global marine productivity (Cullen et al., 2012). In these approaches, approximations are constructed for the relationship between productivity and in situ irradiance weighted for inhibition effectiveness by a set of spectral coefficients, the biological weighting function (BWF). However, no BWFs have been defined for the dominant taxa of the central oceanic regions, either in culture or for natural populations. Moreover, all current BWFs for UV inhibition of photosynthesis have been defined using eukaryotes, and there is evidence that resistance to irradiance stress is less in prokaryotes of the open oligotrophic ocean (Kulk et al., 2011). Early attempts to relate the relative effect of in situ (or simulated in situ) exposure on phytoplankton photosynthesis to broadband irradiance (weighted or unweighted) in temperate to tropical open ocean environments showed on the order of a factor of 2 variation in response at any given exposure (Smith et al., 1980; Behrenfeld et al., 1993). These estimates will presumably be better constrained if more is known about BWFs of the species in these assemblages and how they vary with growth condition.

A primary driver in much of the work on defining the spectral response to UV, in both aquatic and terrestrial environments, has been to assay the effect of variations in short-wavelength UVB coupled to changes in stratospheric ozone (Day and Neale, 2002). Stratospheric ozone was depleted over the latter decades of the 20th century, but continued depletion was halted by controls imposed on the atmospheric release of chlorofluorocarbons (CFCs). Presently, chlorinated species derived from the UV-mediated decomposition of CFCs that have accumulated in the upper stratosphere continue to depress ozone, but the effect is mild in the mid- and low latitudes (Weatherhead and Andersen, 2006). There is little expectation that the very low ozone columns that occur seasonally in high latitudes (where decomposition is enhanced by the catalytic effect of ice crystals) could ever be reached under present or typical future atmospheric conditions in temperate or tropical regions of the ocean where Synechococcus sp. is abundant. However, over longer (geological) timescales episodes of low ozone could occur (or may have already occurred) if the stratosphere is (was) affected by particle showers from a gamma ray burst (Thomas, 2009). Biological weighting functions extending to the short wavelengths that would be highly enhanced under such a scenario will also be useful in estimating the possible impact of a gamma ray burst on marine productivity.

The present contribution reports on the exposure response curves and spectral dependence (BWF) for UV inhibition of photosynthesis in two well-studied strains of Synechococcus sp., generally known by the codes assigned by the Woods Hole Oceanographic Institution, WH7803 and WH8102. The approach is similar to that used in previous studies of the spectral weighting function of phytoplankton photosynthesis, based on a custom spectral incubator, the "photoinhibitron" (Neale and Fritz, 2001). In the present studies, we used a new, expanded version of the photoinhibitron that enhances the estimation of BWF coefficients at short wavelengths. We also present a new model integrating the BWF and the photosynthesis-irradiance curve (BWF/P-E model) that better accounts for the effects of inhibition over an extended range of exposure.

\section{Materials and methods}

\subsection{Growth conditions}

Synechococcus strains CCMP1334 and CCMP2370 (synonymous with WH7803 and WH8102, respectively) were obtained from the National Center for Marine Algae (NCMA, formerly CCMP) and were grown on SN media (Andersen et al., 2005). Growth irradiance was provided by cool white fluorescent lamps on a $14 \mathrm{~h}$ light: $10 \mathrm{~h}$ dark cycle. Growth PAR was measured with a $4-\pi$ probe immersed in water inside a culture flask. The average $( \pm \mathrm{SD})$ of PAR in the growth chamber was $77 \pm 12$ (medium irradiance, or ML) and $174 \pm 22$ (high irradiance, or HL) $\mu \mathrm{mol} \mathrm{m}^{-2} \mathrm{~s}^{-1}$ and $T=20$ and $26^{\circ}$, with semi-continuous dilution. Although these strains can grow at lower temperatures, we chose these temperatures so that they could also be used in parallel experiments with Prochlorococcus, which has a narrower range of growth temperature. Growth curves were measured for each set of culture conditions and experiments were performed with cultures in early-to-mid-log phase. Experiments were repeated at least three times with independently grown cultures for each set of conditions. Cell enumeration was performed with a Multisizer 4 particle sizer/counter (Beckman/Coulter) using a $20 \mu \mathrm{m}$ aperture (minimum resolution $0.4 \mu \mathrm{m}$ ). Samples were diluted as necessary with filtered seawater before counting.

\subsection{Photosynthesis measurements}

Incubations for the measurement of photosynthesis were performed using a polychromatic UV + PAR incubator with $2.5 \mathrm{~kW}$ xenon lamp (photoinhibitron), based on the design of Cullen et al. (1992) with modified block construction similar to that described by Smyth et al. (2012). The present study used six blocks $(16.5 \mathrm{~cm} \times 7 \mathrm{~cm} \times 3.5 \mathrm{~cm})$, each separately plumbed for coolant flow to enhance temperature regulation. Each block has vertical wells (formed by tubes connecting holes on the top and bottom of the block) for $20,1 \mathrm{~cm}$ diameter, quartz bottom, cylindrical cuvettes, arrayed as 4 lengthwise rows of 5 wells. Two filter combinations were used in each block, each covering 10 wells ( 2 lengthwise rows), giving a total of 12 spectral treatments per incubation, with neutral density screens positioned in some slots to produce approximately equal increments of PAR within each treatment. 
Table 1. Filter configuration for the 12 spectral treatment groups of the photoinhibitron as used in this study.

\begin{tabular}{|c|c|c|c|}
\hline \multirow[t]{2}{*}{ Panel* } & \multicolumn{2}{|c|}{$\begin{array}{l}\text { Nominal cutoff } \\
(\% \text { of PAR } T)\end{array}$} & \multirow[t]{2}{*}{ Components } \\
\hline & $1 \%$ & $50 \%$ & \\
\hline A & 268 & 291 & WG280 \\
\hline $\mathrm{B}$ & 285 & 300 & $\mathrm{WG} 280 \times 2^{\mathrm{a}, \mathrm{b}}$ \\
\hline $\mathrm{C}$ & 290 & 300 & WG $280+$ Cellulose Acetate ${ }^{b}$ \\
\hline $\mathrm{D}$ & 283 & 303 & WG305 \\
\hline $\mathrm{E}$ & 290 & 309 & WG305 × 2 \\
\hline $\mathrm{F}$ & 291 & 306 & WG305 + Cellulose Acetate \\
\hline G & 302 & 312 & WG320 \\
\hline $\mathrm{H}$ & 308 & 325 & WG $320 \times 2$ \\
\hline I & 320 & 335 & WG335 \\
\hline $\mathrm{J}$ & 312 & 346 & WG295 + LG350 ${ }^{\mathrm{c}}$ \\
\hline $\mathrm{K}$ & 358 & 375 & WG295 + LG370 \\
\hline $\mathrm{L}$ & 386 & 408 & GG395 + Courtgard $^{\mathrm{d}} \times 2$ \\
\hline
\end{tabular}

\footnotetext{
* Letters identify the panel in Fig. 2 with example results for the listed spectral treatment group.

a All Schott filters were $3 \mathrm{~mm}$ thick; " $\times 2$ " denotes that two pieces of filter glass or film were combined, doubling the effective pathlength (thus red-shifting the cutoff wavelength, especially for $1 \% T$ ).

b All filter combinations (either two pieces of filter glass or filter glass + film as indicated) were assembled with a high-purity silicone optical grease. This decreased variation in the index of refraction along the optical path and minimized scattering. Filter-filter or filter-film combinations had the same transmittance in the visible as single layer elements.

${ }^{c}$ LG filters are manufactured by Corion and contain a polymer film sandwiched between two glass layers. The WG295 "prefilter" protected the polymer film from solarization by the short-wavelength irradiance from the xenon arc lamp.

${ }^{\mathrm{d}}$ Courtgard, a film manufactured by Solutia, Inc, blocks UV below $400 \mathrm{~nm}$.
}

The filter combinations are listed in Table 1. Spectral irradiance $\left(\mathrm{mW} \mathrm{m}^{-2} \mathrm{~nm}^{-1}\right)$ for each well in the photoinhibitron was measured with a custom-built fiber-optic spectroradiometer as described by Neale and Fritz (2001). Some combinations resulted in exposure of suspensions to $E(\lambda)$ at wavelengths $<290 \mathrm{~nm}$, which do not normally reach the ocean surface. These treatments were added to extend model predictions to extremely low ozone columns as would occur if the stratosphere receives particle showers from a gamma ray burst (Thomas, 2009).

Photosynthesis per unit chlorophyll (Chl) $a\left(P^{\mathrm{B}}\right)$ was measured as total ${ }^{14} \mathrm{C}$ assimilation (acid-stable) in $1 \mathrm{~mL}$ aliquots during a $1 \mathrm{~h}$ incubation as described in Sobrino et al. (2007). Chl $a$ was determined fluorometrically on culture aliquots filtered on to GF/F (Whatman) filters. The cells were disrupted by homogenizing the filters in $90 \%$ acetone with a teflon pestle, followed by overnight extraction at $-10^{\circ} \mathrm{C}$. The extracts were clarified by centrifugation and fluorescence was measured before and after acidification on a Turner Designs model AU-10 fluorometer calibrated with Chl $a$ from spinach. Pigment absorbance $\left(a_{\mathrm{p}}(\lambda), \mathrm{m}^{2} \mathrm{mg} \mathrm{Chl}^{-1}\right)$ was measured using the quantitative filter technique (QFT) following the procedures described by Tzortziou et al. (2006).

\subsection{Photosynthesis model}

Data were fit to BWF/P-E functions:

$$
\begin{aligned}
& P^{\mathrm{B}}=P_{\mathrm{s}}^{\mathrm{B}} \cdot\left(1-e^{-E_{\mathrm{PAR}} / E_{s}}\right) \cdot \operatorname{ERC}\left(E_{\mathrm{inh}}^{*}\right) \\
& E_{\mathrm{inh}}^{*}=\sum_{265 \mathrm{~nm}}^{400 \mathrm{~nm}} \varepsilon(\lambda) \cdot E(\lambda) \cdot \Delta \lambda+\varepsilon_{\mathrm{PAR}} \cdot E_{\mathrm{PAR}}
\end{aligned}
$$

where ERC is the exposure response curve for inhibition of photosynthesis. The set of $\varepsilon(\lambda)$ is the BWF (see Table 2 for symbols and units). Given spectral irradiance in each cell, $\varepsilon(\lambda)$ and the other parameters are estimated using a non-linear regression approach. Details of the principalcomponent-based estimation procedure and error assessment are given in Cullen and Neale (1997). Standard errors for the parameter means over-replicate experiments $(n \geq 3)$ are the root mean square (rms, quadrature) of the estimation standard errors (propagated from regression standard errors) and the standard error due to between-replicate variability.

Three different response models (ERCs) were considered for inhibition, differing in how the rate at which activity is restored (referred to as "repair") depends on relative inhibition (referred to as "damage") (Fig. 1). The first ERC considered was the " $E$ " model. This is the original ERC used to model responses to UV as measured in the photoinhibitron (Cullen et al., 1992). It assumes that repair is proportional to damage at all exposures (fixed rate constant for repair), so that at steady-state $P^{\mathrm{B}}$

$$
\frac{P^{\mathrm{B}}}{P_{\mathrm{pot}}^{\mathrm{B}}}=\frac{1}{1+E_{\mathrm{inh}}^{*}} .
$$

In later studies it was observed that the $E$ model was not consistent with UV + PAR inhibition of photosynthesis in a eukaryotic picophytoplankter, Nannochloropsis (Sobrino et al., 2005). For this species, photosynthesis had a steeper decline (greater increase in inhibition) with increased exposure than would be predicted if repair rate scaled with damage for all exposures. This alternative response was better predicted using a fixed repair rate, which is the basis for the " $T$ " model:

$$
\frac{P^{\mathrm{B}}}{P_{\mathrm{pot}}^{\mathrm{B}}}=\left\langle\begin{array}{ll}
1 & E_{\text {inh }}^{*} \leq 1 \\
\frac{1}{E_{\text {inh }}^{*}} & E_{\text {inh }}^{*}>1 .
\end{array}\right.
$$

The " $T$ " designation relates to the presence of a threshold $\left(E_{\mathrm{inh}}^{*}=1\right)$ above which, by definition, photosynthesis is inhibited. Compared to the $E$ model, this model was significantly better at fitting observations at high $E_{\text {inh }}^{*}$ in several studies of cultures and natural assemblages (Sobrino and Neale, 2007; Sobrino et al., 2005; Sobrino et al., 2009). However, the imposition of a threshold in the $T$ model has the disadvantage of ignoring the modest, but still evident, effects of UV and PAR at low exposures. In this study we introduce a new model that can account for responses at both low and 

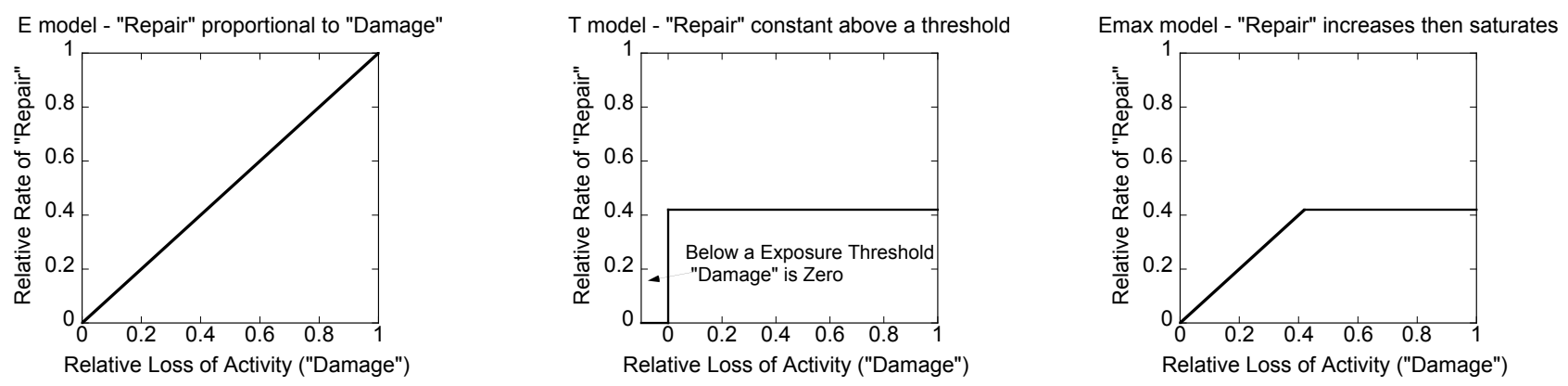

Figure 1. Graphical illustration of the implied dependence of repair and damage rates for the $E, T$ and $E_{\max }$ models.

Table 2. Notation.

\begin{tabular}{|c|c|c|}
\hline Symbol & Name & Units \\
\hline$a_{\mathrm{p}}(\lambda)$ & Phytoplankton chlorophyll-specific spectral absorption coefficient & $\mathrm{m}^{2} \mathrm{mg} \mathrm{Chl}^{-1}$ \\
\hline$a_{\mathrm{IS}}(\lambda)$ & Irradiance-weighted chlorophyll-specific absorption of PAR at depth $z$ & $\mathrm{~m}^{2} \mathrm{mg} \mathrm{Chl}^{-1}$ \\
\hline$a_{\text {PI }}$ & Irradiance-weighted chlorophyll-specific absorption of PAR in the photoinhibitron & $\mathrm{m}^{2} \mathrm{mg} \mathrm{Chl}^{-1}$ \\
\hline BWF & Biological weighting function & \\
\hline$c$ & Scaling factor for exposures $>E_{\max }$ & dimensionless \\
\hline$\varepsilon(\lambda)$ & Biological weight of inhibitory effect of UV & $\mathrm{m}^{2} \mathrm{~mW}^{-1}$ \\
\hline$\varepsilon_{\mathrm{PAR}}$ & Biological weight of inhibitory effect of PAR & $\mathrm{m}^{2} \mathrm{~W}^{-1}$ \\
\hline$E_{\text {inh }}^{*}$ & Irradiance weighted for effectiveness in inhibiting photosynthesis & dimensionless \\
\hline$E(\lambda)$ & UV irradiance at wavelength $\lambda=265-400 \mathrm{~nm}$ & $\mathrm{~mW} \mathrm{~m}^{-2} \mathrm{~nm}^{-1}$ \\
\hline$E_{\max }$ & Exposure that saturates repair rate & dimensionless \\
\hline$E_{\mathrm{PAR}}$ & PAR irradiance $(400-700 \mathrm{~nm})$ & $\mathrm{W} \mathrm{m}^{-2}$ \\
\hline$E_{\mathrm{PAR}}(z)$ & Underwater PAR irradiance at depth $z$ & $\mathrm{~W} \mathrm{~m}^{-2}$ \\
\hline$E_{\mathrm{PAR}}^{\prime}(z)$ & $\begin{array}{l}\text { Underwater PAR irradiance at depth } z \text { adjusted for difference in pigment } \\
\text { absorption of PAR in situ vs. in the photoinhibitron }\end{array}$ & $\mathrm{W} \mathrm{m}^{-2}$ \\
\hline & Characteristic irradiance for onset of saturation of photosynthesis & $\mathrm{W} \mathrm{m}^{-2}$ \\
\hline$\left.E^{Q_{(0}}-, \lambda\right)$ & Spectral photon flux density of PAR $(400-700 \mathrm{~nm})$ at the sea surface & $\mu \mathrm{mol}$ photons $\mathrm{m}^{-2} \mathrm{~s}^{-1}$ \\
\hline$E_{\mathrm{PI}}^{Q}(\lambda)$ & Spectral photon flux density of PAR $(400-700 \mathrm{~nm})$ in the photoinhibitron & $\mu \mathrm{mol}$ photons $\mathrm{m}^{-2} \mathrm{~s}^{-1}$ \\
\hline$P_{\mathrm{S}}^{\mathrm{B}}$ & Biomass-normalized photosynthetic rate & $\mathrm{mgCmgChl}-1 \mathrm{~h}^{-1}$ \\
\hline$P_{\text {pot }}^{\mathrm{B}}$ & Biomass-normalized potential photosynthetic rate in absence of inhibition & $\mathrm{mgC} \mathrm{mg} \mathrm{Chl}{ }^{-1} \mathrm{~h}^{-1}$ \\
\hline$P_{\mathrm{S}}^{\mathrm{B}}$ & Irradiance-saturated (maximum) photosynthetic rate in absence of inhibition & $\mathrm{mg} \mathrm{Cmg} \mathrm{Chl}{ }^{-1} \mathrm{~h}^{-1}$ \\
\hline
\end{tabular}

high exposures. This model is called the " $E_{\max }$ " model and uses a combination of the $E$ model at low exposures and $T$ model at high exposures:

$$
\begin{aligned}
\frac{P^{\mathrm{B}}}{P_{\mathrm{pot}}^{\mathrm{B}}} & =\left\langle\begin{array}{ll}
1 /\left(1+E_{\mathrm{inh}}^{*}\right) & E_{\mathrm{inh}}^{*} \leq E_{\mathrm{max}}^{*} \\
1 / c E_{\mathrm{inh}}^{*} & E_{\mathrm{inh}}^{*}>E_{\max }^{*}
\end{array}\right. \\
c & =\frac{1+E_{\max }^{*}}{E_{\max }^{*}} .
\end{aligned}
$$

The new $E_{\max }$ parameter defines the transition between the exposure range over which repair rate increases with damage and higher exposures for which repair rate is constant (i.e., operating at some maximum rate). A related, timedependent, version of the model, the $R_{\max }$ model, has been used previously to fit the UV + PAR inhibition of photosynthesis in phytoplankton from the Ross Sea, Antarctica (Smyth et al., 2012).
Each of these models has an implied relationship between damage and repair that is illustrated in Fig. 1. The $E_{\max }$ model has a greater range of possible response shapes that is gained through an additional parameter compared to the $E$ or $T$ model. Whether sufficient increase in explained variance is gained to justify the incorporation of an additional parameter was assessed by evaluation of the Akaike information criterion (AIC; Andersen et al., 2005) for each of the fits using the Matlab NonLinearModel function (Statistics toolbox).

\subsection{Predicting depth profiles of photosynthesis}

For an initial evaluation of model performance under quasireal-world conditions, example profiles of photosynthesis were calculated using a set of nominal estimates of downwelling spectral irradiance in the oligotrophic ocean. Spectral irradiance just under the surface, $E^{\mathrm{Q}}\left(0^{-}, \lambda\right)$, and diffuse 
attenuation coefficients, $K_{\mathrm{d}}(\lambda)$, were estimated using the methods of Cullen et al. (2012), using their provided worksheet for estimation of bio-optical data. Input parameters were $23^{\circ} \mathrm{N}$ latitude, 21 June noon, $0.1 \mathrm{mg} \mathrm{Chl} \mathrm{m}^{-3}$ and $0.01 \mathrm{~m}^{-1} \mathrm{CDOM}$ (colored dissolved organic matter) absorption at $400 \mathrm{~nm}$. Photosynthesis rates at each depth point, $z$, were then computed following a procedure similar to that described in Lehmann et al. (2004), with some modifications. The model photosynthetic response is a function of total PAR $\left(E_{\mathrm{PAR}}, \mathrm{W} \mathrm{m}^{-2}\right)$. A factor is applied to underwater PAR to correct for spectral differences of model PAR vs. the filtered xenon irradiance used to measure photosynthesis. Irradianceweighted chlorophyll-specific absorption for the photoinhibitron, $a_{\mathrm{PI}}\left(\mathrm{m}^{2} \mathrm{mg} \mathrm{Chl}{ }^{-1}\right)$, was calculated by weighting the phytoplankton chlorophyll-specific spectral absorption coefficient $\left(a_{\mathrm{p}}(\lambda), \mathrm{m}^{2} \mathrm{mg} \mathrm{Chl}^{-1}\right)$ with the average photoinhibitron spectrum, as photon flux, $E_{\mathrm{PI}}^{\mathrm{Q}}(\lambda)$ :

$a_{\mathrm{PI}}=\sum_{400 \mathrm{~nm}}^{700 \mathrm{~nm}} a_{\mathrm{p}}(\lambda) \cdot E_{\mathrm{PI}}^{\mathrm{Q}}(\lambda) \cdot \Delta \lambda / \sum_{400 \mathrm{~nm}}^{700 \mathrm{~nm}} E_{\mathrm{PI}}^{\mathrm{Q}}(\lambda) \cdot \Delta \lambda$.

The calculation was based on photon (quantum) flux $\left(E^{\mathrm{Q}}\right)$ since photosynthesis is a quantum process. The wavelength resolution $(\Delta \lambda)$ was $1 \mathrm{~nm}$. A similar calculation was performed for the underwater profile to obtain the irradiance-weighted absorption of in situ irradiance, $a_{\mathrm{IS}}(z)$ $\left(\mathrm{m}^{2} \mathrm{mg} \mathrm{Chl}^{-1}\right)$ :

$$
\begin{aligned}
a_{\mathrm{IS}}(z)= & \sum_{400 \mathrm{~nm}}^{700 \mathrm{~nm}} a_{\mathrm{p}}(\lambda) \cdot E^{\mathrm{Q}}\left(0^{-}, \lambda\right) \cdot e^{K_{\mathrm{d}}(\lambda) \cdot z} \cdot \Delta \lambda / \\
& \sum_{400 \mathrm{~nm}}^{700 \mathrm{~nm}} E^{\mathrm{Q}}\left(0^{-}, \lambda\right) \cdot e^{K_{\mathrm{d}}(\lambda) \cdot z} \cdot \Delta \lambda .
\end{aligned}
$$

Finally, a corrected PAR irradiance for the photosynthesis model was calculated as

$$
E_{\mathrm{PAR}}^{\prime}(z)=E_{\mathrm{PAR}}(z) \frac{a_{\mathrm{IS}}}{a_{\mathrm{PI}}} .
$$

For consistency with previous usage of the BWF/P-E model, the corrected PAR is in energy units. This correction adjusted $E_{\mathrm{PAR}}$ for the greater (or lesser) absorption of underwater irradiance compared to photoinhibitron irradiance; this applied to both light-limited photosynthesis at low irradiance and PAR inhibition at high irradiance (pigments mediate both processes). Next, profiles of total weighted irradiance for inhibition $\left(E_{\mathrm{inh}}^{*}\right)$ were calculated:

$$
\begin{aligned}
E_{\mathrm{inh}}^{*}(z) & =\sum_{280 \mathrm{~nm}}^{400 \mathrm{~nm}} \varepsilon(\lambda) \cdot E^{\mathrm{Q}}\left(0^{-}, \lambda\right) \cdot e^{K_{\mathrm{d}}(\lambda) \cdot z} \cdot \Delta \lambda \\
& +\varepsilon_{\mathrm{PAR}} \cdot E_{\mathrm{PAR}}^{\prime}(z) ;
\end{aligned}
$$

and productivity at depth was obtained by evaluating Eq. (1) with the $E_{\max } \operatorname{ERC}$ (Eq. 4).

\section{Results}

\subsection{Photosynthesis}

A representative set of photosynthesis measurements obtained using the photoinhibitron as configured for this study is shown in Fig. 2. The rate of photosynthesis over a $1 \mathrm{~h}$ incubation is plotted versus the PAR irradiance, each panel showing results obtained with one of the 12 filter combinations used in the experiment. Each filter combination controls the minimum wavelength of exposure ("cutoff") and the shape of the spectrum near the cutoff. The position of each cell within the projected beam of the xenon arc lamp also affects the spectral shape as well as the total irradiance. Further variation in total irradiance was configured using neutral density screens. Since both spectral composition and irradiance can vary even within one spectral group, the observations do not necessarily follow a smooth response vs. exposure trend (e.g., circled points in Fig. 2). However, the variation in spectral shape is taken into account in the fitting of the BWF given the treatment irradiance spectrum separately measured for each cell. To illustrate this, Fig. 2 also shows the corresponding predicted values from the fitted $\mathrm{BWF}_{E_{\max }} / \mathrm{P}-\mathrm{E}$ model (x's, overall $R^{2}=0.96$ and root mean square error $(\mathrm{RMSE})=0.53 \mathrm{mg} \mathrm{C} \mathrm{mg} \mathrm{Chl}^{-1} \mathrm{~h}^{-1}$ ), which closely follow the observed variation both between treatments and within each treatment group.

\subsection{Selection of exposure response curve}

We tested how well each of three possible ERCs fit the response of Synechococcus to UV + PAR exposure. All three models were fit for each photoinhibitron experiment. An example set of photoinhibitron results using the responses of a WH8102 culture (grown at $\mathrm{HL}$ and $26^{\circ} \mathrm{C}$ ) is shown in Fig. 3, where photosynthesis $\left(P^{B}\right)$ is plotted versus weighted irradiance $\left(E_{\mathrm{inh}}^{*}\right.$, dimensionless). Fits using all of the ERCs reproduced the general variation within the data set, with an $\mathrm{R}^{2}$ $>0.90$ (RMSE $<0.7 \mathrm{mg} \mathrm{C} \mathrm{mg} \mathrm{Chl}{ }^{-1} \mathrm{~h}^{-1}$ ). However, there were small but significant differences in the performance of the ERCs. When a BWF was estimated with the $E$ model, there was good agreement between observed (points) and predicted (line) biomass-specific photosynthesis at low exposure, but the best-fit model systematically overestimated photosynthesis at high exposure (Fig. $3-E$ model). In comparison, the predicted photosynthesis from the BWF/P-E using the $T$ model (Fig. $3-T$ model) was closer than the $E$ model predictions at high exposure but systematically underestimated photosynthesis at low exposure. This motivated the application of the hybrid model $\left(E_{\max }\right)$ that uses an $E$ model response at low exposure and a $T$ model response at high exposure (see model description in Materials and methods). The $E_{\max }$ model gave a better overall fit to the data than either the $E$ or $T$ model, although response in all cases was still consistently overestimated when inhibition was $>80 \%$ 

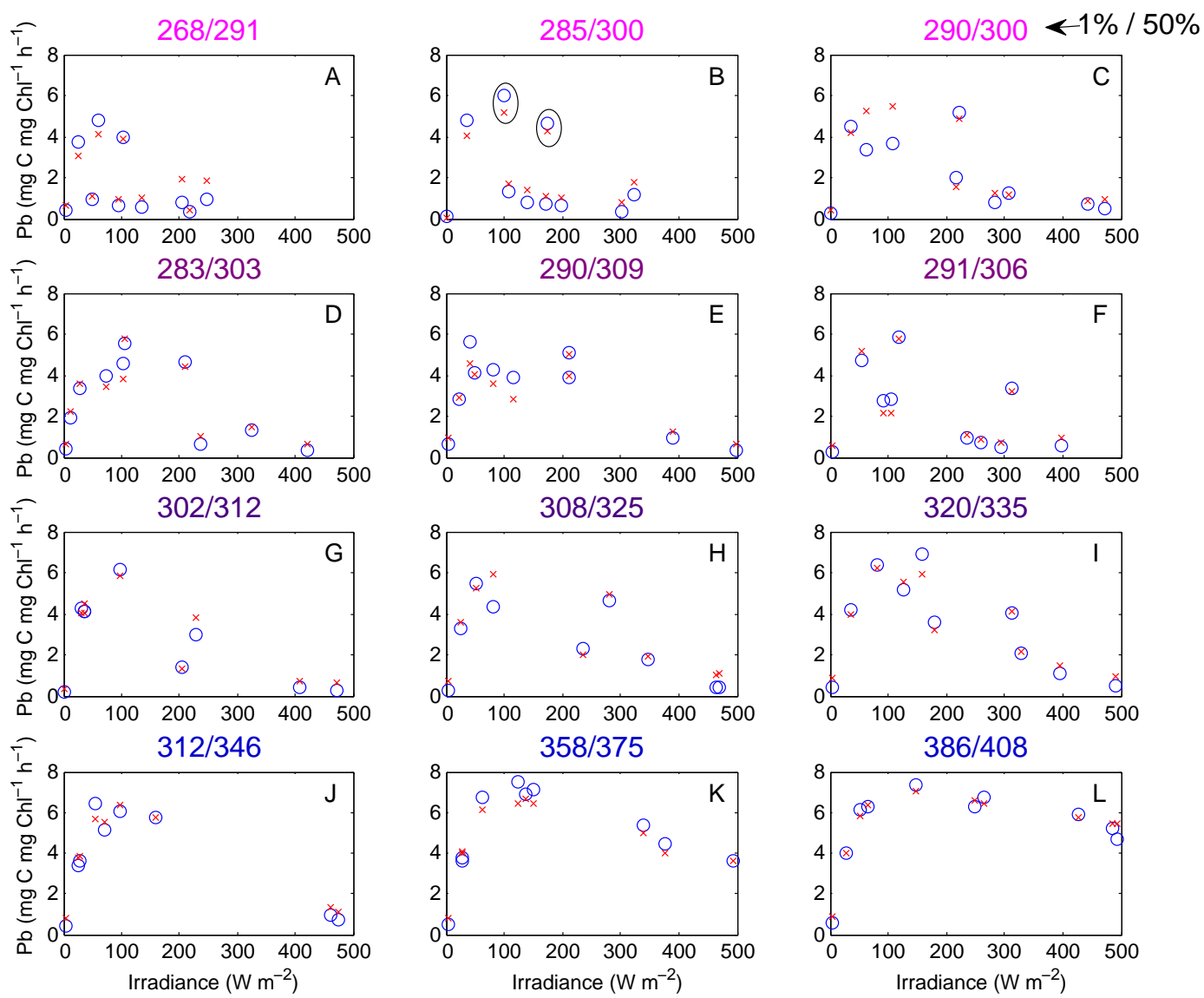

$320 / 335$
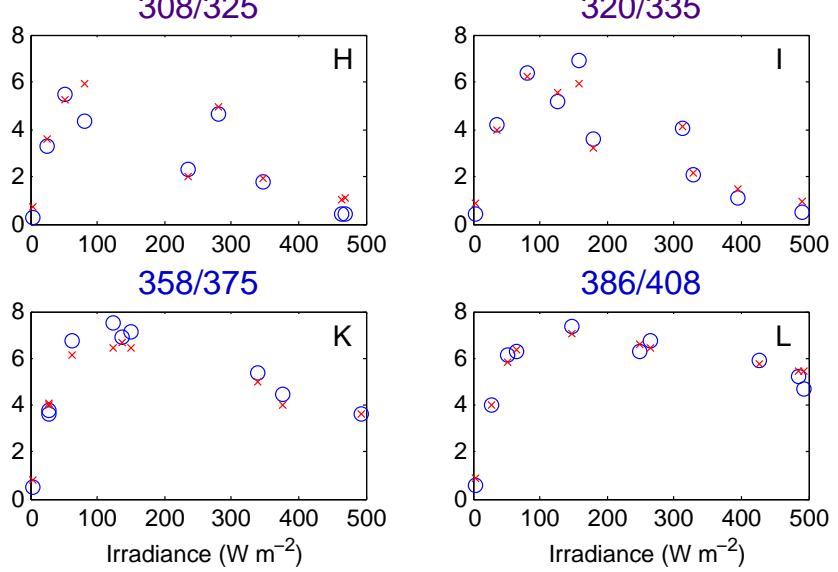

Figure 2. Representative set of photosynthesis measurements from a Synechococcus photoinhibitron experiment; shown are results from the exposure of a HL $26^{\circ} \mathrm{C}$ WH8102 culture plotted vs. PAR exposure (W m ${ }^{-2}$ ) of each treatment. Observed rate of photosynthesis (open circles) and predicted rate of phytosynthesis by the $\mathrm{BWF}_{E_{\max }}-\mathrm{PE}$ model (x's). Panel titles give the 1 and $50 \%$ wavelength cutoffs of the spectral treatment shown in each panel, color coded on a gradient from short-wavelength UVB (magenta) to long-wavelength UVA (blue); further details are listed by panel letter in Table 1. Due to heterogeneity in the Xe lamp emission, spectral composition varies somewhat even within a spectral treatment. This causes some scatter in the P-E relationship (e.g., circled points) but does not affect the generally good agreement between observed and fitted. The root mean square error (RMSE) for the fit is $0.53\left(\mathrm{mg} \mathrm{C} \mathrm{mg} \mathrm{Chl}{ }^{-1} \mathrm{~h}^{-1}\right)$.

(Fig. $3-E_{\max }$ model). The average $R^{2}$ was 0.96 and 0.95 and average RMSE was 0.37 and $0.40\left(\mathrm{mg} \mathrm{C} \mathrm{mg} \mathrm{Chl}^{-1} \mathrm{~h}^{-1}\right)$ for WH7803 and WH8102 cultures, respectively. To assess whether the improved fit from the $E_{\max }$ model was sufficient to justify an additional parameter, we computed the AIC for each fit. The results confirmed that the additional parameter was justified by the improved predictive accuracy of the $E_{\max }$ model. The AIC takes into account both the prediction performance and number of model parameters; the best model is the one providing the lowest AIC (Andersen et al., 2005). Consistent with the examples in Fig. 3, the AIC was lower for the $T$ vs. $E$ model fit, and for the $E_{\max }$ vs. the $T$ model. Although the improvement varied from experiment to experiment (averages presented in Table 3), the AIC consistently decreased for all experiments with WH8102 $(n=15)$ and WH7803 $(n=16)$, except for one WH8102 experiment where the $T$ model and $E_{\max }$ model AIC were essentially the same (AIC was 0.6 higher for $E_{\max }$ ).

\subsection{Spectral range of the biological weighting function}

In most experiments previously conducted with the photoinhibitron, the spectral treatments were defined with 8 different long-pass cutoff filters, and the shortest wavelength included in the exposure was in the 281-290 nm range (e.g., Neale and Fritz, 2001). Generally the irradiance at wavelengths $<300 \mathrm{~nm}$ was very small, though this is in line with solar irradiance. Moreover, because treatments with shortest wavelength cutoffs also have very high levels of UVA that usually cause considerable inhibition, the response to the additional UVB is relatively small. Thus, these treatments do not have much leverage in the overall fit. In contrast, for this study we were particularly interested in having more statistical power to estimate coefficients at the short wavelengths to maximize our ability to assess the impact of a drastic decrease in ozone as would occur with a gamma ray burst. 


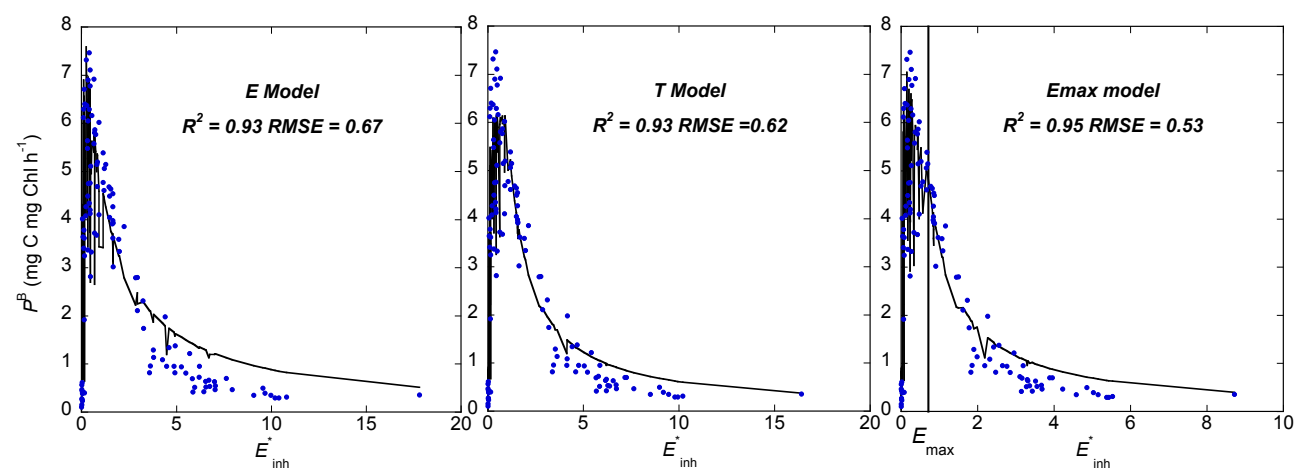

Figure 3. Observed (points) and fitted (lines) biomass-specific photosynthesis $\left(\mathrm{mg} \mathrm{C} \mathrm{mg} \mathrm{Chl}^{-1}\right)$ as a function of UV $+\mathrm{PAR}$ exposure weighted by a spectral biological weighting function for inhibition, $E_{\mathrm{inh}}^{*}$ (dimensionless). The panels illustrate the observed vs. fitted results for three BWF/P-E models, the $E$ (left), $T$ (center) and $E_{\max }$ (right) models. RMSE is in $\mathrm{mg} \mathrm{C} \mathrm{mg} \mathrm{Chl}{ }^{-1} \mathrm{~h}^{-1}$. The fitted value for $E_{\mathrm{max}}$ is shown by the vertical line in the panel on the right. Observations are for an exposure in the photoinhibitron of a $\mathrm{HL} 26^{\circ} \mathrm{C}$ culture of WH8102 (same as Fig. 2).

Table 3. Difference in AICs calculated for $E, T$ and $E_{\text {max }}$ model fits to experimental data on the response of Synechococcus photosynthesis to UV + PAR exposure. The comparison is limited to experiments conducted after a $1 \mathrm{~h}$ pre-exposure of culture sample to moderate UV + PAR (see Sect. 2).

\begin{tabular}{llrr}
\hline Strain & Measure & $\Delta \mathrm{AIC}$ & $\begin{array}{r}\Delta \mathrm{AIC} \\
(T \text { vs. } E)\end{array}$ \\
& & $\left(E_{\max }\right.$ vs. $\left.T\right)$ \\
\hline \multirow{2}{*}{ WH8102 } & Average & -38.6 & -30.2 \\
& SD & 18.5 & 18.6 \\
WH7803 & Average & -64.8 & -12.2 \\
& SD & 21.3 & 10.1 \\
\hline
\end{tabular}

To increase statistical power in the short UV-B, the number of spectral treatments was increased to 12 and the minimum treatment wavelength extended to $265 \mathrm{~nm}$ (cf. Table 1). To assess how this change in experimental design affected the estimation of the BWF, we performed fits both with the full data set and a reduced-size data set that had a similar spectral range as previous fits with eight spectral treatments. The reduced data set omitted the two treatments with spectral irradiance below $282 \mathrm{~nm}$ (filter combinations A and B in Table 1), with the total number of photosynthesis observations reduced from 120 to 100 . The BWFs estimated using the principal components method were very similar (identical within the standard error of the estimates) for the wavelength range 300 to $400 \mathrm{~nm}$ (Fig. 4). However, below $300 \mathrm{~nm}$ the BWF estimates diverged. When the BWF was fit with the full data set, the general log-linear slope of the BWF in the UV-B above $300 \mathrm{~nm}$ continues approximately the same below $300 \mathrm{~nm}$. In contrast, in the reduced data set, the loglinear slope steepened below $300 \mathrm{~nm}$.

This suggests that the steeper slope in the reduced data set is an "edge" artifact related to the application of the PCA estimation method. Near the wavelength lower limit, both

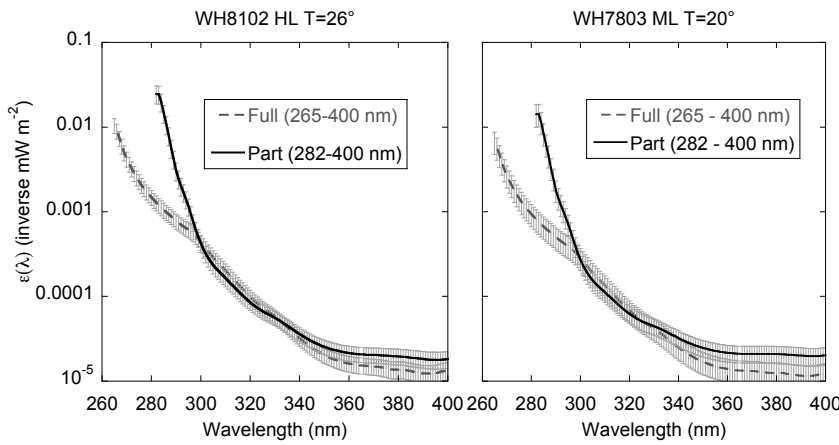

Figure 4. Fitted biological weighting functions ( \pm standard error) for UV inhibition of photosynthesis in Synechococcus WH8102 $\left(\mathrm{HL} 26^{\circ} \mathrm{C}\right.$ ) and $7803\left(\mathrm{ML} 20^{\circ} \mathrm{C}\right.$ ) comparing results obtained using all the data from each experiment ("full", $n=120$, shortest treatment wavelength $265 \mathrm{~nm}$ ) and a reduced data set, omitting the two treatments with spectral irradiance shorter than $282 \mathrm{~nm}$ ("part", $n=100$ ).

the mean and standard deviation (SD) of treatment (photoinhibitron) irradiance approaches zero. Since the reciprocal SD is a scaling factor in the BWF calculation (Cullen and Neale, 1997), the decline in SD at the short-wavelength edge forces high weights irrespective of their actual effect. The overestimation is a structural bias (as opposed to being due to experimental error) since the estimated standard error (which is conditioned on having identified the correct model) is small. The statistical leverage of data for which this bias results in a loss of fit must be small, as essentially the same $R^{2}$ was obtained for the model fit to the $n=100$ data set with either BWF (for BWFs in Fig. 4, difference in $R^{2}$ is ca. 0.002 ; results not shown). Similarly, the artifact has a nearly negligible effect on model predictions under solar exposures (in the absence of a gamma ray burst) since wavelengths $<300 \mathrm{~nm}$ make a very small contribution to $E_{\mathrm{inh}}^{*}$ (cf. Sobrino et al., 2009). In the BWF fit to the full 
data set, an analogous steepening of the BWF can be observed for wavelengths $<280 \mathrm{~nm}$. This is likely caused by the same edge artifact in the PCA method, shifted to $20 \mathrm{~nm}$ shorter wavelengths due to the inclusion of treatments with the shorter cutoffs. Since wavelengths shorter than $280 \mathrm{~nm}$ are extremely unlikely to reach the surface of the ocean in any significant amount, even in the presence of a gamma ray burst, only weighting coefficients for $280 \mathrm{~nm}$ and above are presented herein.

\subsection{Effect of pre-exposure}

We tested the effect of recent light history on photosynthetic response to UV by conducting incubations with cultures that had been pretreated by $1 \mathrm{~h}$ exposure to moderately high PAR $\left(400 \mu \mathrm{mol} \mathrm{m}{ }^{-2} \mathrm{~s}^{-1}\right)$ and UV from a xenon source filtered to exclude wavelengths $<350 \mathrm{~nm}$. These results were compared to those obtained for cultures that were transferred directly from the growth chamber to the photoinhibitron. The latter experiments were performed first, and used to choose the pretreatment conditions so as to cause minimal or low inhibition $\left(E_{\mathrm{inh}}^{*}<E_{\max }\right)$. The photosynthetic rates of samples without pre-exposure (Fig. 5 left panel) exhibited relatively low rates at high UV, below even the best-fit $E_{\max }$ predictions, whereas the pretreated samples (Fig. 5 right panel) had increased photosynthesis and showed a better fit to the $E_{\max }$ model. In addition, the fitted value for $E_{\max }$ increased from $0.32 \pm 0.16$ without pre-exposure to $0.73 \pm 0.24$ with preexposure, consistent with an increase in repair capacity. This implies that pretreatment increased resistance to UV exposure, an interpretation that is also consistent with the kinetic data of WH8102 during moderate UV + PAR exposure as reported by Fragoso et al. (2013). They observed that effective quantum yield of photosystem II (PSII) during UV + PAR exposure dropped to an initial low steady state followed by a "rebound" in yield over the next 20 min period. Kinetic analysis showed that the rebound was consistent with an increase in repair rate.

\subsection{Model fits for different growth conditions}

Maximum rates of uninhibited photosynthesis $\left(P_{\mathrm{s}}^{\mathrm{B}}\right)$ and saturation irradiance parameter $\left(E_{\mathrm{S}}\right)$ were higher for cultures grown at higher temperature (Table 4 ). There were no consistent differences in these parameters for the two different irradiance conditions, except for $\mathrm{WH} 8102,26^{\circ} ; E_{\mathrm{S}}$ was higher for cultures grown under $\mathrm{HL}$ vs. $\mathrm{ML}$, and $P_{\mathrm{s}}^{\mathrm{B}}$ was higher, but the difference was not significant within the variability of the measurements. The parameter for inhibition by $E_{\mathrm{PAR}}, \varepsilon_{\mathrm{par}}$, also tended to be lower for cultures grown at higher irradiance, but not significantly so.

Average BWFs \pm SE $(n \geq 3)$ for WH8102 and WH7803 cultures grown at two irradiance levels and two temperatures are shown in Fig. 6. The two strains showed different patterns of response to changes in growth irradiance and temperature.

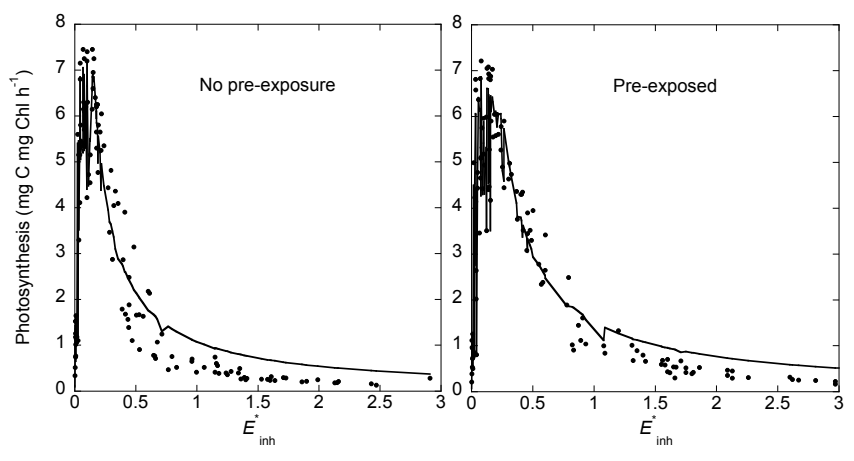

Figure 5. Observed (points) and fitted (lines) photosynthesis of Synechococcus as a function of weighted UV + PAR exposure for two cultures (both WH8102 ML $26^{\circ} \mathrm{C}$ ), for which, prior to a standard $1 \mathrm{~h}$ incubation in the photoinhibitron, one was maintained under growth conditions and the other exposed to moderate UV + PAR (no pre-exposure or pre-exposed, respectively; see details in Materials and methods). Solid line shows the fitted values from the $\mathrm{BWF}_{E_{\max }} / \mathrm{P}-\mathrm{E}$ model.

For WH8102, the variation in average BWF with growth irradiance or temperature was small relative to the standard error of the mean. Differences bordered on significant in the UVA, with lower sensitivity at the higher irradiance and temperature (Fig. 6 upper panels). On the other hand for WH7803, growth irradiance had a strong effect on the BWF at $20^{\circ} \mathrm{C}$, and weak effect at $26^{\circ} \mathrm{C}$, and on average, weights were much lower for cultures grown at the higher temperature (Fig. 6 lower panels).

The variation in the $E_{\max }$ parameter complemented the variation in weights. For WH8102, $E_{\max }$ was greater at the higher temperature for ML, and was slightly increased for HL. For 7803, $E_{\max }$ was, in contrast, lower at the higher temperature at ML, with the same, but not significant, trend for HL-grown cultures (Table 4).

\subsection{Prediction of in situ profiles of photosynthesis}

Both spectral weight $(\varepsilon(\lambda))$ and $E_{\max }$ affect the overall sensitivity to inhibition, so comparisons of responses between strains or between growth conditions need to take both parameters into account. To provide a context for assessing the combined impact of variation in the UV response and P-E parameters, we performed trial calculations of the depth profile of photosynthesis given the fitted $\mathrm{BWF}_{E_{\max }} / \mathrm{P}-\mathrm{E}$ obtained for each strain and growth condition, and blue water bio-optics as described in Sect. 2.3 (Fig. 7). The calculations used average phytoplankton spectral absorption coefficients for each culture condition; these results are not shown as they are similar to those in the literature (e.g., Six et al., 2004). Since the in situ spectrum is efficiently absorbed by Synechococcus pigments, spectrally corrected PAR penetrates deeper than $100 \mathrm{~m}$, and the depth at which $E_{\mathrm{PAR}}^{\prime}(z)$ decreases to $1 \%$ of $E_{\mathrm{PAR}}^{\prime}(0)$ is $>120 \mathrm{~m}$. Thus, the model predicts significant 
Table 4. Fitted parameters for the $E_{\max }$ model, mean \pm standard errors for $n \geq 3$ experiments under each condition.

\begin{tabular}{|c|c|c|c|c|c|c|c|}
\hline \multirow[b]{2}{*}{ Strain } & \multicolumn{2}{|c|}{ Growth conditions } & \multirow[b]{2}{*}{$P_{\mathrm{s}}^{b}$} & \multirow[b]{2}{*}{$E_{\mathrm{S}}$} & \multirow[b]{2}{*}{$\varepsilon_{\text {par }} \times 10^{-3}$} & \multirow[b]{2}{*}{$E_{\max }$} & \multirow[b]{2}{*}{$\int P_{z}^{\mathrm{B}} / \int P_{\mathrm{pot}}^{B}{ }^{*}$} \\
\hline & Light & $T^{\circ} \mathrm{C}$ & & & & & \\
\hline \multirow[t]{4}{*}{ WH8102 } & ML & 20 & $4.20 \pm 0.28$ & $25.5 \pm 3.0$ & $1.52 \pm 1.45$ & $0.49 \pm 0.11$ & $0.72 \pm 0.03$ \\
\hline & & 26 & $5.43 \pm 0.55$ & $34.9 \pm 2.4$ & $1.11 \pm 1.03$ & $0.73 \pm 0.14$ & $0.77 \pm 0.02$ \\
\hline & HL & 20 & $3.11 \pm 0.37$ & $25.4 \pm 2.4$ & $0.32 \pm 0.65$ & $0.46 \pm 0.07$ & $0.83 \pm 0.01$ \\
\hline & & 26 & $6.45 \pm 1.14$ & $39.5 \pm 2.9$ & $0.97 \pm 0.90$ & $0.57 \pm 0.13$ & $0.80 \pm 0.01$ \\
\hline \multirow[t]{4}{*}{ WH7803 } & ML & 20 & $3.83 \pm 0.50$ & $16.9 \pm 1.5$ & $2.20 \pm 1.16$ & $0.54 \pm 0.10$ & $0.71 \pm 0.03$ \\
\hline & & 26 & $8.01 \pm 1.55$ & $29.5 \pm 2.5$ & $0.67 \pm 1.01$ & $0.23 \pm 0.10$ & $0.81 \pm 0.03$ \\
\hline & HL & 20 & $3.65 \pm 0.97$ & $16.1 \pm 2.9$ & $0.89 \pm 0.70$ & $0.35 \pm 0.07$ & $0.81 \pm 0.02$ \\
\hline & & 26 & $7.23 \pm 0.80$ & $28.8 \pm 2.4$ & $0.57 \pm 0.61$ & $0.26 \pm 0.08$ & $0.83 \pm 0.01$ \\
\hline
\end{tabular}

* Integral productivity predicted for test profile conditions (Fig. 7), ratio of prediction for full UV + PAR exposure to potential productivity (inhibition term excluded).

rates of production at depths of $100-150 \mathrm{~m}$. It should be kept in mind that these depths are normally below the thermocline and no attempt has been made to correct for changes in photosynthetic response related to the depth-dependent decreases in temperature and photoacclimation to lower growth irradiance. Nevertheless, the profile calculations are useful to compare the overall responses implicit in the fitted models.

Average biomass-specific productivity varied by a factor of three among the different parameter sets. The magnitude of the variation is mainly driven by the similar range in variation of $P_{\mathrm{s}}^{\mathrm{B}}$ with growth temperature. In addition, the overall photosynthetic performance (productivity relative to uninhibited potential rate) was better at the higher temperature (Table 4, last column). The enhancement was most pronounced for ML-grown cultures. Another manifestation of the greater effect of inhibition on cultures grown at lower temperature is that the depth of the peak was consistently deeper; i.e., the effect of near-surface inhibition extended further down into the water column. Generally, predicted responses were more variable for WH7803 than WH8102.

Although the effect of near-surface exposure differed among the parameter sets, in every case UV + PAR inhibition was predicted to depress a major fraction of water column productivity. Integrated production (to $150 \mathrm{~m}$ ) was $71 \%$ of the potential for the most sensitive case (WH7803, $20^{\circ} \mathrm{C}, \mathrm{ML}$ ) and $83 \%$ of potential for the least sensitive case (WH7803, $26^{\circ} \mathrm{C}, \mathrm{HL}$ ), corresponding to an inhibition (1 relative performance) of $17-29 \%$. The range was smaller for WH8102. Predicted inhibition under surface exposure $(1 \mathrm{~m})$ varied between 91 and $78 \%$ (average response for the most and least sensitive case, respectively; cf. Fig. 8). These calculations were performed using spectral irradiance modeled with an ozone column of 300 Dobson units (DU), climatological for the Northern Hemisphere midlatitude summer (Lamsal et al., 2004). Decreasing ozone column to 200 DU, in the range of what would occur in a gamma ray burst (Thomas, 2009), resulted in a small decrease in surface rates, between 0.7 and $1.5 \%$ relative to the uninhibited rate. Because the rates at the surface are already low, this is a 6.0 to $7.6 \%$ decrease below surface productivity predicted under the normal ozone column (300 DU). Nevertheless, these effects are confined to the near-surface zone so that integrated productivity is predicted to be at most $0.3 \%$ lower under the lower-ozone column.

To put these responses in the context of previous studies of UV + PAR inhibition effects on phytoplankton photosynthesis, we predicted productivity under the same set of trial conditions using a $\mathrm{BWF}_{E_{\max }} / \mathrm{P}$-E model fitted from data for the common coastal/estuarine species Thalassiosira pseudonana. Calculations were performed based on experiments using cultures (strain $3 \mathrm{H}$ ) grown under conditions similar to the $20^{\circ} \mathrm{C}$, ML conditions used here (data from Sobrino et al., 2009). These observations were refit to the $\mathrm{BWF}_{E_{\max }} / \mathrm{P}-\mathrm{E}$ model for the purpose of comparisons (results not shown). Using this fitted model, we predicted for both T. pseudonana and Synechococcus the profile of (1) potential productivity (no inhibition), (2) productivity including only PAR inhibition and (3) productivity predicted by the full model with both UV and PAR inhibition (Fig. 8). For Synechococcus we show the results for $20^{\circ} \mathrm{C}$, ML cultures averaged over all experiments with WH8102 and WH7803. Under these conditions, the diatom and Synechococcus have similar Chlspecific productivity in the absence of inhibition. However both PAR and UV + PAR inhibition are appreciably more severe for Synechococcus. This is evident from both the lower rates at the surface and the slower increase of productivity with depth for Synechococcus. The difference in response to UV contributes the most to the contrast. Performance over the water column for full spectral exposure was $71 \%$ of potential production for Synechococcus compared to $83 \%$ for T. pseudonana. 


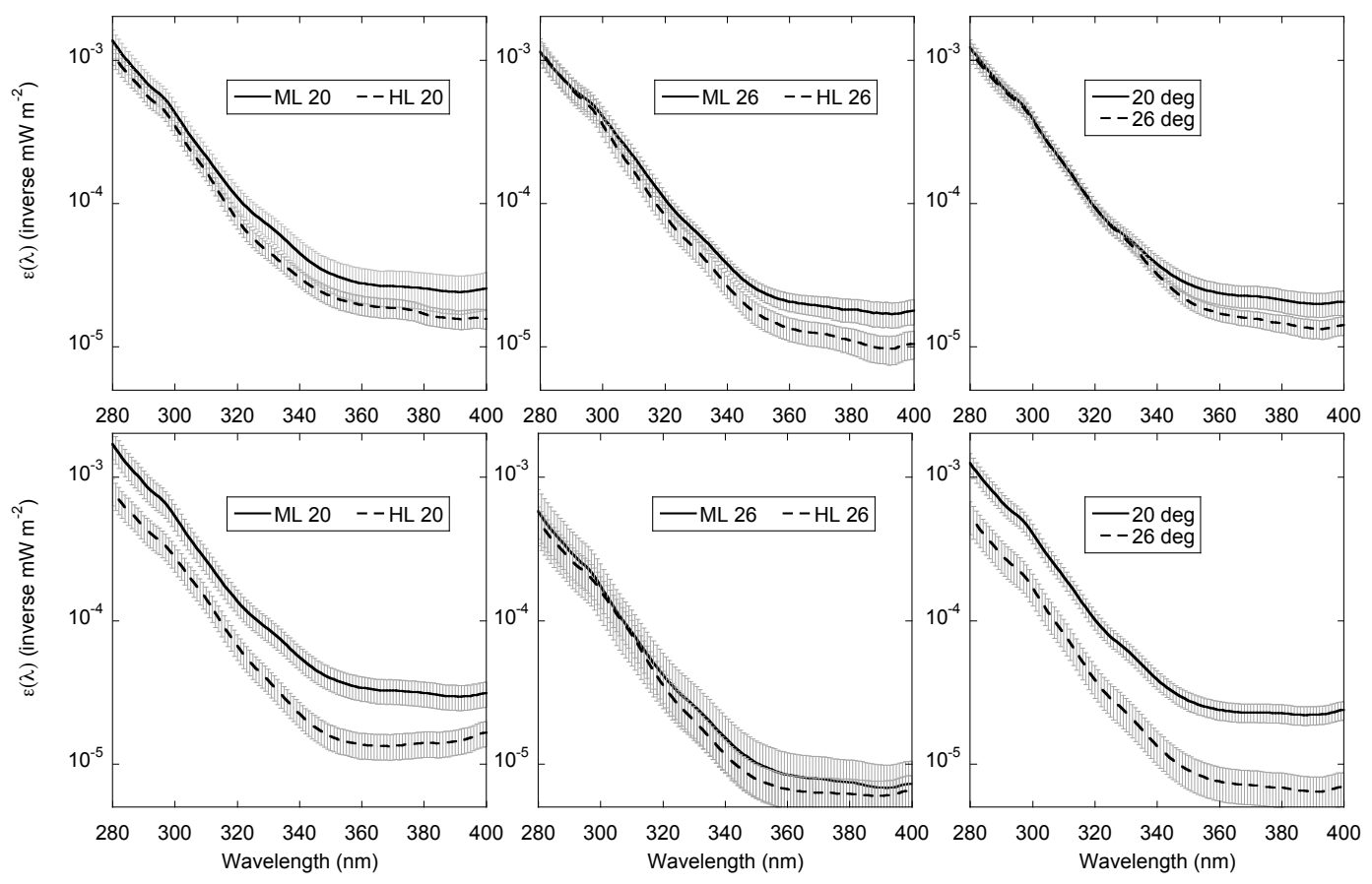

Figure 6. Average ( \pm standard error) of biological weighting functions for WH8102 (upper three panels) and WH7803 (lower three panels) grown in either "medium" irradiance (ML, $77 \mu \mathrm{mol} \mathrm{m} \mathrm{m}^{-2} \mathrm{~s}^{-1} \mathrm{PAR}$ ) or "high" irradiance (HL, $174 \mu \mathrm{mol} \mathrm{m}{ }^{-2} \mathrm{~s}^{-1}$ PAR), and growth temperatures of 20 or $26^{\circ} \mathrm{C}$. Weights were estimated for $265-400 \mathrm{~nm}$, but the weights for wavelengths below 280 nm are not shown since coefficients in the $265-280 \mathrm{~nm}$ range may be influenced by the edge artifact illustrated in Fig. 4.

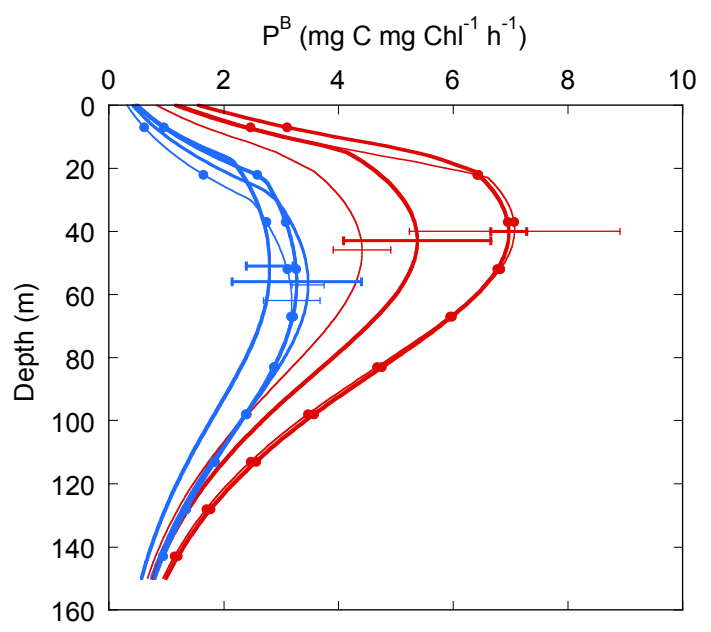

Figure 7. Predicted profiles of biomass-specific productivity $\left(P^{\mathrm{B}}\right)$ for a test set of water column optical conditions (noon, summer solstice, $15^{\circ} \mathrm{S}$ latitude, oligotrophic bio-optics, etc.) based on fitted $\mathrm{BWF}_{E_{\max }} / \mathrm{P}-\mathrm{E}$ models estimated from experiments with Synechococcus strains WH8102 (lines with no symbols) and WH7803 (lines with circles) for each of four growth conditions, $20^{\circ} \mathrm{C}$ (blue), $26^{\circ} \mathrm{C}$ (red), medium PAR (thin line), high PAR (thick line). The line is the average profile $(n=3)$ for replicate experiments; horizontal bars indicate simple standard deviation of $P^{\mathrm{B}}$ estimates from replicate experiments at the depth of peak average productivity.

\section{Discussion}

Photosynthesis by both of the studied Synechococcus strains was strongly inhibited by UV exposure. These strains were both originally isolated from surface waters but are considered representative of assemblages from different oceanic regions (Six et al., 2007a). Different clades of Synechococcus strains have been defined based on genomic sequence (Scanlan et al., 2009). WH8102 has been classified as a member of clade III, which is most common in oligotrophic regions. It has a characteristically high ratio of phycouribilin to phycoerythobilin (PUB : PEB), which maximizes light absorption in the blue (Scanlan, 2003). WH7803 is a member of clade $\mathrm{V}$, a more generalist clade, which has a low PUB : PEB ratio enabling light absorption over wavelengths characteristic of both oligotrophic and coastal waters.

Response to high PAR and UV of both strains was dependent on the growth conditions. Sensitivity to UV, e.g., as measured by the UV associated decrease in model predicted in situ production, was reduced when cultures were grown at the higher temperature. Higher growth irradiance was also associated with lower sensitivity. Similar dependence of sensitivity to UV inhibition of photosynthesis on growth conditions has been reported for other laboratory studies of phytoplankton (Litchman and Neale, 2005; Sobrino and Neale, 2007). The sensitivity of PSII quantum yield to UV exposure in WH7803 was also less in high-light-grown 


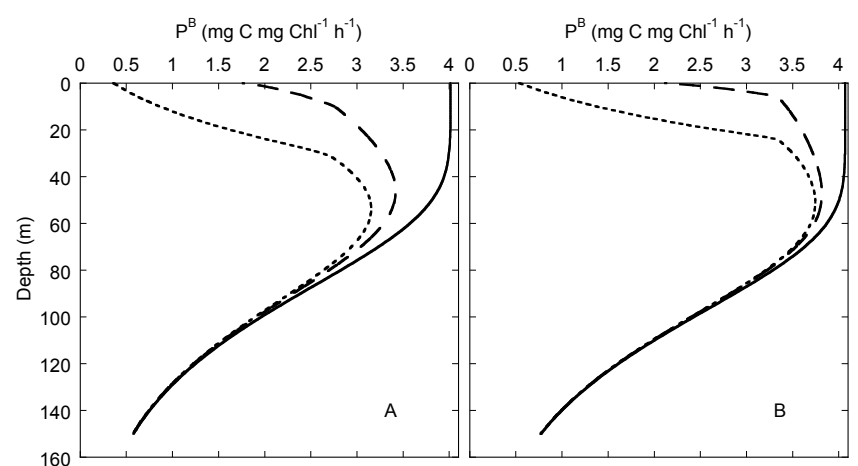

Figure 8. Depth profiles of productivity as predicted using the $\mathrm{BWF}_{E_{\max }} / \mathrm{P}-\mathrm{E}$ model evaluated with the P-E equation and no inhibition (solid line), with only inhibition by PAR $(\varepsilon$ PAR $)$ included (long dashes) or with full UV + PAR inhibition (short dashes). The curves in (A) show the average response by Synechococcus (ML $20^{\circ}$ cultures, WH8102 and WH7803 combined); in (B) is shown predicted response based on a fit of the $\mathrm{BWF}_{E_{\max }} / \mathrm{P}-\mathrm{E}$ model to the photoinhibitron data for the diatom Thalassiosira pseudonana (strain $3 \mathrm{H}$ ) grown at comparable growth irradiance and temperature (from Sobrino et al., 2009).

versus medium- or low-light-grown cultures (Garczarek et al., 2008). Changes in sensitivity were reflected in differences in the fitted BWF, $E_{\max }$ and inhibition by PAR $\left(\varepsilon_{\mathrm{PAR}}\right)$. Interestingly, while overall sensitivity showed similar trends for both strains, the pattern of changes in the model parameters was different between the strains. For WH8102, the lower sensitivity was mainly caused by an increase in $E_{\text {max }}$. There was some shift in the BWF towards smaller weights, but this shift was small relative to the inherent variability between replicate cultures (Fig. 6). In contrast for WH7803, $E_{\max }$ was actually somewhat lower for hightemperature/high-light cultures. Unlike WH8102, BWF coefficients decreased significantly with higher growth irradiance in $20^{\circ}$ cultures, and the average BWF for highertemperature cultures was several-fold lower than that of lower-temperature cultures (Fig. 6).

These results suggest that WH8102 and WH7803, which have different pigment configurations, also differ in how photoacclimation influences the response to inhibitory exposure. Reducing sensitivity to UV can be accomplished by increasing photoprotection (thus decreasing damage) and/or increasing repair (Banaszak, 2003; Neale, 2000). By definition, the weighting coefficients in the $\mathrm{BWF}_{E_{\max }} / \mathrm{P}-\mathrm{E}$ model represent a ratio of the rate constants of damage and repair. A rate constant is a measure of the likelihood that a given UV-susceptible target is damaged and/or reactivated per unit time. On the other hand, $E_{\max }$ is a measure of repair capacity that is related to what proportion of target sites are damaged when repair rate reaches an upper limit. WH8102 showed large changes in $E_{\max }$ suggesting that part of its photoadaptation strategy is to increase repair capacity. This apparent increase in repair capacity over growth timescales is interest- ing given that WH8102 also appears to increase repair over shorter timescales (minutes-hour) in response to acute UV exposure (Fragoso et al., 2013). Fragoso et al. (2013) suggest that this could be related to increased expression of a more UV resistant isoform of the PSII reaction center core protein, D1. Other than the change in $E_{\max }$ between growth conditions for WH8102, there was little change in the BWF, and overall a narrower acclimation range than estimated for WH7803 (e.g., based on extent of differences in relative profile performance between growth conditions). The more constrained acclimation behavior of WH8102 would be consistent with its overall genomic characterization of having less regulatory genes (e.g., kinases) than other cyanobacteria, thought to be related to its association with more constant oligotrophic environments (Palenik et al., 2003).

For WH7803, BWF coefficients were variable between growth conditions that could occur through changes in rate constants of damage (e.g., increased photoprotection), rate constants of repair or a change in both of these rate constants. Unlike WH8102, $E_{\max }$ decreased with HL or highertemperature growth, which may seem to be inconsistent with the overall decline in sensitivity. However, $E_{\max }$ more precisely represents the point of transition between a scaled (= rate constant $\times$ number of damage sites) and a constant rate of repair (Fig. $1-E_{\max }$ model). The decrease in $E_{\max }$ (e.g., between 20 and $26^{\circ}$ cultures) could thus be due to a greater increase in the rate constant of repair than repair capacity. This is equivalent to a steeper initial slope of function in Fig. 1, and there will be a corresponding lowering of the fraction needed to reach a fixed repair capacity (transition point moves closer to origin). This suggests that WH7803 has (relative to WH8102) a more diverse repertoire of acclimation to irradiance and temperature, manifested in its variable sensitivity to inhibition by UV irradiance. This is consistent with the distribution of the clade V group to which WH7803 belongs over a broad range of oligotrophic and coastal environments (Scanlan et al., 2009).

Overall, the $E_{\max }$ model clearly provided the best prediction of inhibition of photosynthesis in both studied strains of Synechococcus. Together with previous studies using the " $T$ " model (Sobrino et al., 2005; Sobrino and Neale 2007; Sobrino et al., 2009), these results suggest that there is an upper limit to repair rate in many phytoplankton during exposure to high UV + PAR. The physiological mechanism(s) limiting absolute repair rates is (are) presently not known and more work is needed. The dynamics of photosynthetic complexes involved in photoinhibition and recovery, particularly PSII, has received considerable study (recent reviews: Vass, 2012, and Tyystjärvi, 2013) and lead to development of models of PSII damage and repair (Campbell and Tyystjärvi, 2012). However, these models presently assume a fixed rate constant for repair (equivalent to the $E$ model). Nevertheless, it is also recognized that repair, e.g., of PSII, is a multistep process involving degradation of damaged components, their resynthesis and reintegration into a reactivated complex 
(recent review: Takahashi and Murata, 2008). The rate of any of these steps, or a step in the repair of another complex such as RUBISCO, could reach a maximum under high exposure and set the upper limit of repair rate. Furthermore, there is increasing evidence that repair itself can be inhibited under irradiance stress (Takahashi and Murata, 2008). Such inhibition could account for the tendency of even the $E_{\max }$ model to over-estimate photosynthesis at very high exposure (cf. Fig. 3).

Our results suggest that the primary productivity of open ocean Synechococcus will be significantly depressed in the near-surface, "photoactive" zone, with most of the effect induced by the UV portion of the spectrum. Presently, there are few field data to compare with the predictions of the $\mathrm{BWF}_{E_{\max }} / \mathrm{P}-\mathrm{E}$ model. Early studies of UV inhibition of photosynthesis in open ocean assemblages (e.g., Behrenfeld et al., 1993; Smith et al., 1980) pre-dated the routine use of flow-cytometric techniques to quantitate the contribution of Synechococcus. Nevertheless, the range of relative inhibition reported in those studies encompasses the inhibition obtained in the example calculations presented here. More recently, rates of photosynthesis have been reported for in situ incubations of a Synechococcus-dominated assemblage in the Coral Sea $\left(22^{\circ} \mathrm{S}\right)$, comparing containers transmitting full spectrum, UVA + PAR and PAR only (Conan et al., 2008). Again, the results of these near-surface, $6 \mathrm{~h}$ incubation results are broadly consistent with our example calculations, with the full spectrum rate at $1 \mathrm{~m} 35 \%$ of the PAR-only rate, and the UVA + PAR rate $53 \%$ of the PAR-only rate. Currently, a more comprehensive effort is underway to estimate productivity based on the $\mathrm{BWF}_{E_{\max }} / \mathrm{P}$-E model over a range of latitudes and time using representative oceanic observations from the Pacific Ocean to make more specific comparisons, including a more thorough evaluation of possible gamma ray burst effects; these results will be reported in a future contribution. Also, the UV responses of the typical co-dominant of Synechococcus in the picoplankton, Prochlorococcus, have also been studied using laboratory cultures and will be presented in a separate report.

Our study is the first report of the spectral dependence of inhibition for photosynthesis by marine prokaryotic (pico)phytoplankton. Overall, the strains used in this study were more sensitive to inhibition than a eukaryotic nanoplankton, Thalassiosira pseudonana, grown under similar conditions. The sensitivity is also high in the context of the large-scale analysis of Cullen et al. (2012), who examined the relative decrease in integrated productivity based on selected BWFs (all for eukaryotic nano/microplankton) under a wide range of conditions. Their maximum inhibition was $24 \%$, which was exceeded by the responses of the ML $20^{\circ}$ cultures (28\% average inhibition of integrated productivity under the test conditions). The higher sensitivity of Synechococcus occurred despite the demonstrated existence of mechanisms protecting against and recovering from high light exposure (Bailey and Grossman, 2008). Neverthe- less, the relatively higher sensitivity of Synechococcus vs. T. pseudonana is consistent with a general trend of greater resistance to light stress and faster photoacclimation for eukaryotic vs. prokaryotic phytoplankton grown under the same conditions (Kulk et al., 2011). While UV sensitivity has been extensively studied for eukaryotic phytoplankton, further studies are needed to confirm the generality of high sensitivity to UV for picocyanobacteria, especially spectral and temporal dependence.

Acknowledgements. This research was supported by NASA grant NNX09AM85G to Brian Thomas, Patrick J. Neale and Adrian Melott. The authors thank Glaucia Fragoso for assistance with incubations and helpful comments on the manuscript.

Edited by: E. Boss

\section{References}

Andersen, R. A., Berges, J. A., Harrison, P. J., and Watanabe, M. Recipes for Freshwater and Seawater Media, in: Algal Culturing Techniques, edited by: Andersen, R. A., Elsevier Academic Press, Hong Kong, 429-538, 2005.

Bailey, S. and Grossman, A.: Photoprotection in Cyanobacteria: Regulation of Light Harvesting, Photochem. Photobiol., 84, 1410-1420, doi:10.1111/j.1751-1097.2008.00453.x, 2008.

Banaszak, A. T.: Photoprotective physiological and biochemical responses of aquatic organisms, in: UV effects in aquatic organisms and ecosystems, edited by: Helbling, E. W. and Zagarese, H. E., Comprehensive Series in Photosciences, Royal Society of Chemistry, Cambridge, UK, 329-356, 2003.

Behrenfeld, M., Hardy, J., Gucinski, H., Hanneman, A., Lee, H. I., and Wones, A.: Effects of ultraviolet-B radiation of primary production along latitudinal transects in the South Pacific ocean, Mar. Environ. Res., 35, 349-363, 1993.

Campbell, D. A. and Tyystjärvi, E.: Parameterization of photosystem II photoinactivation and repair, Biochim. Biophys. Acta, 1817, 258-265, doi:10.1016/j.bbabio.2011.04.010, 2012.

Conan, P., Joux, F., Torréton, J. P., Pujo-Pay, M., Douki, T., Rochelle-Newall, E., and Mari, X.: Effect of solar ultraviolet radiation on bacterio- and phytoplankton activity in a large coral reef lagoon (southwest New Caledonia), Aquat. Microb. Ecol., 52, 83-98, 2008.

Cullen, J. J., Neale, P. J., and Lesser, M. P.: Biological weighting function for the inhibition of phytoplankton photosynthesis by ultraviolet radiation, Science, 258, 646-650, 1992.

Cullen, J. J. and Neale, P. J.: Biological weighting functions for describing the effects of ultraviolet radiation on aquatic systems, in: Effects of ozone depletion on aquatic ecosystems, edited by: Häder, D.-P. and Landes, R. G., Austin, 97-118, 1997.

Cullen, J. J., Davis, R. F., and Huot, Y.: Spectral model of depthintegrated water column photosynthesis and its inhibition by ultraviolet radiation, Global Biogeochem. Cy., 26, GB1011, doi:10.1029/2010gb003914, 2012.

Day, T. A. and Neale, P. J.: Effects of UV-B radiation on terrestrial and aquatic primary producers, Anun. Rev. Ecol. Syst., 33, 371396, 2002. 
Fichot, C. G. and Miller, W. L.: An approach to quantify depthresolved marine photochemical fluxes using remote sensing: Application to carbon monoxide (CO) photoproduction, Remote Sens. Environ., 114, 1363-1377, doi:10.1016/j.rse.2010.01.019, 2010.

Fragoso, G. M., Neale, P. J., Kana, T. M., and Pritchard, A. L.: Kinetics of Photosynthetic Response to Ultraviolet and Photosynthetically Active Radiation in Synechococcus WH8102 (CYANOBACTERIA), Photochem. Photobiol., 90, 522-32, doi:10.1111/php.12202, 2013.

Garczarek, L., Dufresne, A., Blot, N., Cockshutt, A. M., Peyrat, A., Campbell, D. A., Joubin, L., and Six, C.: Function and evolution of the psbA gene family in marine Synechococcus: Synechococcus sp. WH7803 as a case study, ISME J., 2, 937-953, 2008.

Harrison, J. W. and Smith, R. E. H.: Effects of ultraviolet radiation on the productivity and composition of freshwater phytoplankton communities, Photochem. Photobiol. Sci., 8, 1218-1232, 2009.

Kulk, G., de Poll, W. H. v., Visser, R. J. W., and Buma, A. G. J.: Distinct differences in photoacclimation potential between prokaryotic and eukaryotic oceanic phytoplankton, J. Exp. Mar. Biol. Ecol., 398, 63-72, doi:10.1016/j.jembe.2010.12.011, 2011.

Lamsal, L. N., Weber, M., Tellmann, S., and Burrows, J. P.: Ozone column classified climatology of ozone and temperature profiles based on ozonesonde and satellite data, J. Geophys. Res., 109, D20304, doi:10.1029/2004JD004680, 2004.

Lehmann, M. K., Davis, R. F., Huot, Y., and Cullen, J. J.: Spectrally weighted transparency in models of water-column photosynthesis and photoinhibition by ultraviolet radiation, Mar. Ecol.-Prog. Ser., 260, 101-110, 2004.

Litchman, E. and Neale, P. J.: UV Effects on photosynthesis, growth and acclimation of an estuarine diatom and cryptomonad, Mar. Ecol.-Prog. Ser., 300, 53-62, 2005.

Mella-Flores, D., Six, C., Ratin, M., Partensky, F., Boutte, C., Le Corguillè, G., Blot, N., Gourvil, P., Kolowrat, C., Garczarek, L., and Marie, D.: Prochlorococcus and Synechococcus have evolved different adaptive mechanisms to cope with light and UV stress, Front. Microbiol., 3, 285, doi:10.3389/fmicb.2012.00285, 2012.

Neale, P. J.: Spectral weighting functions for quantifying the effects of ultraviolet radiation in marine ecosystems, in: The effects of UV radiation in the marine environment, edited by: de Mora, S. J., Demers, S., and Vernet, M., Environmental Chemistry Series, Cambridge Univ. Press, Cambridge, 72-100, 2000.

Neale, P. J. and Fritz, J. J.: Experimental exposure of plankton suspensions to polychromatic ultraviolet radiation for determination of spectral weighting functions, in: Ultraviolet Groundand Space-based Measurements, Models, and Effects, edited by: Slusser, J., Herman, J. R., and Gao, W., Aerospace, Remote Sensing, and Astronomy, SPIE-The International Society for Optical Engineering, San Diego, 291-296, 2001.

Palenik, B., Brahamsha, B., Larimer, F. W., Land, M., Hauser, L., Chain, P., Lamerdin, J., Regala, W., Allen, E. E., McCarren, J., Paulsen, I., Dufresne, A., Partensky, F., Webb, E. A., and Waterbury, J.: The genome of a motile marine Synechococcus, Nature, 424, 1037-1042, 2003.

Scanlan, D. J.: Physiological diversity and niche adaptation in marine Synechococcus, Adv. Microbial Physiol., 47, 1-64, 2003.

Scanlan, D. J., Ostrowski, M., Mazard, S., Dufresne, A., Garczarek, L., Hess, W. R., Post, A. F., Hagemann, M.,
Paulsen, I., and Partensky, F.: Ecological Genomics of Marine Picocyanobacteria, Microbiol. Mol. Biol. R., 73, 249-299, doi:10.1128/mmbr.00035-08, 2009.

Six, C., Thomas, J. C., Brahamsha, B., Lemoine, Y., and Partensky, F.: Photophysiology of the marine cyanobacterium Synechococcus sp. WH8102, a new model organism, Aquat. Micro. Ecol., 35, 17-29, 2004.

Six, C., Finkel, Z. V., Irwin, A. J., and Campbell, D. A.: Light Variability Illuminates Niche-Partitioning among Marine Picocyanobacteria, PLoS ONE, 2, e1341, doi:10.1371/journal.pone.0001341, 2007a.

Six, C., Joubin, L., Partensky, F., Holtzendorff, J., and Garczarek, L.: UV-induced phycobilisome dismantling in the marine picocyanobacterium Synechococcus sp. WH8102, Photosynth. Res., 92, 75-86, 2007b.

Smith, R. C., Baker, K. S., Holm-Hansen, O., and Olson, R. S.: Photoinhibition of photosynthesis in natural waters, Photochem. Photobiol., 31, 585-592, 1980.

Smyth, R. L., Sobrino, C., Phillips-Kress, J., Kim, H.-C., and Neale, P. J.: Phytoplankton photosynthetic response to solar ultraviolet irradiance in the Ross Sea Polynya: Development and evaluation of a time-dependent model with limited repair, Limnol. Oceanogr., 57, 1602-1618, 2012.

Sobrino, C., Neale, P. J., and Lubian, L.: Interaction of UVRadiation and Inorganic Carbon Supply in the Inhibition of Photosynthesis: Spectral and Temporal Responses of Two Marine Picoplankters, Photochem. Photobiol., 81, 384-393, 2005.

Sobrino, C. and Neale, P. J.: Short-term and long-term effects of temperature on phytoplankton photosynthesis under UVR exposures, J. Phycol., 43, 426-436, 2007.

Sobrino, C., Neale, P. J., Phillips-Kress, J. D., Moeller, R. E., and Porter, J.: Elevated $\mathrm{CO}_{2}$ increases sensitivity to ultraviolet radiation in lacustrine phytoplankton assemblages, Limnol. Oceanogr., 54, 2448-2459., 2009.

Takahashi, S. and Murata, N.: How do environmental stresses accelerate photoinhibition?, Trends Plant Sci., 13, 178-182, 2008.

Thomas, B. C.: Gamma-ray bursts as a threat to life on Earth, Int. J. Astrobiol., 8, 183-186, doi:10.1017/s1473550409004509, 2009.

Tyystjärvi, E.: Chapter Seven - Photoinhibition of Photosystem II, in: International Review of Cell and Molecular Biology, edited by: Kwang, W. J., Academic Press, 243-303, 2013.

Tzortziou, M., Herman, J. R., Gallegos, C. L., Neale, P. J., Subramaniam, A., Harding, L. W., and Ahmad, Z.: Bio-optics of the Chesapeake Bay from measurements and radiative transfer closure, Estuar. Coast. Shelf Sci., 68, 348-362, 2006.

Vass, I.: Molecular mechanisms of photodamage in the Photosystem II complex, Biochimica et Biophysica Acta (BBA) - Bioenergetics, 1817, 209-217, doi:10.1016/j.bbabio.2011.04.014, 2012.

Villafañe, V., Sundbäck, K., Figueroa, F., and Helbling, E.: Photosynthesis in the aquatic environment as affected by UVR, in: UV effects in aquatic organisms and ecosystems, edited by: Helbling, E. W. and Zagarese, H. E., Comprehensive Series in Photosciences, Royal Society of Chemistry, Cambridge, UK, 357-397, 2003.

Weatherhead, E. C. and Andersen, S. B.: The search for signs of recovery of the ozone layer, Nature, 441, 39-45, 2006. 